\title{
Minimization of Hesitant L-fuzzy Automaton
}

\author{
${ }^{* 1}$ Marzieh Shamsizadeh, ${ }^{2}$ Mohammad Mehdi Zahedi, Mohamad Javad Agheli Goki \\ ${ }^{1}$ Department of Mathematics, Behbahan Khatam Alanbia University of Technology, Khouzestan, Iran \\ Email: shamsizadeh.m@gmail.com \\ ${ }^{2}$ Department of Mathematics, Graduate University of Advanced Technology, Kerman, Iran \\ Email:zahedi_mm@kgut.ac.ir \\ ${ }^{2}$ Department of Mathematics, Graduate University of Advanced Technology, Kerman, Iran \\ Email:mjavadagheli@gmail.com
}

\begin{abstract}
In this paper, we study a new generalization for the notion of fuzzy automata, which we called hesitant Lfuzzy automaton (HLFA). We present the formulations of the mathematics framework for the theory of HLFA. Moreover, we present the concepts of hesitant L-fuzzy behavior and inverse hesitant L-fuzzy behavior recognized by a type of HLFA. After that, for any hesitant L-fuzzy language we present a minimal complete accessible deterministic hesitant L-fuzzy automaton recognizing that. Finally, we present an algorithm, which determines states of the minimal hesitant L-fuzzy automaton and we present the time complexity of the algorithm.
\end{abstract}

\section{Keywords: Hesitant automata, Hesitant set, Behavior, Lattice}

\section{Introduction}

The theory of fuzzy sets was introduced by L.A. Zadeh in 1965 [22]. Recently, the concept of hesitant fuzzy set was introduced by Torra and Narukawa [19, 20]. Their approach permits the membership values of elements to be an arbitrary subset of $[0,1]$. This concept can react to the human's hesitancy more objectively than the other classical extension of fuzzy sets. In other words, hesitant fuzzy sets are quite suitable for the situation where we have a set of possible values rather than a margin of error or some possibility distribution on the possible values. Observe that hesitant fuzzy sets can be seen as multi-fuzzy sets, i.e., sets of fuzzy sets. Inspired by Goguen's approach, Dehmiry, Mashinchi and Mesiar [5] extends the valuation set of hesitant fuzzy sets to a lattice rather than $[0,1]$ and introduces hesitant $L$-fuzzy sets.

W.G.Wee [21] introduced the idea of fuzzy automata. E.T. Lee and L.A. Zadeh [9] in 1969 gave the concept of fuzzy finite state automata. Fuzzy finite automata have many important applications [7, 10, 13, 14, 15, 16, 17, 18].

Recently, Costa and Bedregal in [4] using the concepts of typical hesitant fuzzy set [19,2] introduced the concept of nondeterministic typical hesitant fuzzy automaton and presented a subclass, called deterministic typical hesitant fuzzy automaton, which generalizes the notion of deterministic finite automaton.

\footnotetext{
${ }^{*}$ Corresponding author; E-mail: shamsizadeh.m@gmail.com
} 
State minimization is a fundamental problem in automata theory. There are many papers on the minimization problem of fuzzy finite automata. For example, minimization of mealy type of fuzzy finite automata in discussed in [3], minimization of fuzzy finite automata with crisp final states without outputs in studied in [1], minimizing the deterministic finite automaton with fuzzy (final) states in [12]. Myhill-Neroded's theorem has been extended to fuzzy regular language and also an algorithm is given for minimizing the deterministic finite automaton with fuzzy (final) states in $[8,12]$.

In this paper, the theory of hesitant L-fuzzy automaton will be presented, characterizing the behaviors and languages computed by hesitant L-fuzzy automata. This work has the following division, first this introduction, then in Section 2, presents all the basic definitions and notations. Section 3, presents the notion of hesitant L-fuzzy automaton (HLFA) and hesitant L-fuzzy behavior (HLFB). After that, some of the closure properties for the HLFA are considered. Moreover, we present the concepts of HLFB and inverse hesitant L-fuzzy behavior recognized by a type of HLFA. Also, we show that if $\beta_{1}$ and $\beta_{2}$ are two HLFBs, then $\beta_{1} \sqcup \beta_{2}$ is so. Section 4, gives a congruence relation by considering the HLFB, and by using the equivalence classes presents an HLFA recognized HLFB. Section 5, for any hesitant L-fuzzy language $A$, we present a minimal complete deterministic hesitant L-fuzzy automaton recognizing $A$. Also, we present an algorithm to determines states of the minimal hesitant L-fuzzy automaton with time complexity of $O\left(|Q|^{2}|X|^{|Q|-1}\right)$.

\section{Preliminaries}

In this section, we present all the basic definitions and notations that are used throughout the text.

Definition 1. [5] Let $X$ denotes a universal set and $\left(L, \preceq_{L}\right)$ be an arbitrary lattice. Then a hesitant L-fuzzy set of $X$ is a mapping

$$
\begin{gathered}
H: X \rightarrow 2^{L} \\
x \mapsto H(x) .
\end{gathered}
$$

The value $H(x)$ denotes the membership degree of $x$ in $H$ and is named hesitant L-fuzzy element ( $H L F E$ ).

Definition 2. [5] For $A, B \in 2^{L}$, the function $\sqcup: 2^{L} \times 2^{L} \rightarrow 2^{L}$ is computed by:

$$
A \sqcup B=\{a \vee b \mid a \in A \text { and } b \in B\},
$$

and the function $\sqcap: 2^{L} \times 2^{L} \rightarrow 2^{L}$ is computed by:

$$
A \sqcap B=\{a \wedge b \mid a \in A \text { and } b \in B\} .
$$

Definition 3. [5] Let $(L, \preceq)$ be an arbitrary lattice and $S$ be a real valued non-constant function such that

$$
\begin{gathered}
S: L \rightarrow R \\
a \mapsto S(a) .
\end{gathered}
$$

The function $\mathrm{S}$ is said a score function on $L$ if the following condition is satisfied:

$$
A_{1} \preceq A_{2} \Rightarrow S\left(A_{1}\right) \leq S\left(A_{2}\right),
$$

for every $A_{1}, A_{2} \in L$. 
Definition 4. [5] Let $C_{s}$ be a function on $L$ such that $C_{s}: L \rightarrow L$ such that

$$
\begin{aligned}
C_{s}: L & \rightarrow L \\
& A \mapsto C_{s}(A)
\end{aligned}
$$

The function $C_{s}$ is said complement function on $L$ based on score function $S$ if the following conditions satisfied:

1. Global boundary condition: $C_{S}(\vee L)=\wedge L, C_{S}(\wedge L)=\vee L$.

2. Local boundary condition: $C_{S}(\vee A)=\wedge\left(C_{s}(A)\right), C_{S}(\wedge A)=\vee\left(C_{s}(A)\right)$, for every $A \in L$.

3. Monotonicity: if $S\left(A_{1}\right)<S\left(A_{2}\right)$, then $S\left(C_{S}\left(A_{1}\right)\right)>S\left(C_{S}\left(A_{2}\right)\right)$, for every $A_{1}, A_{2} \in L$.

4. $C_{S}\left(C_{S}(A)\right)=A$, for every $A \in L$.

Definition 5. [5] The complement of an $H \in H L F E$ related to a score function is defined as follows:

$$
H^{c}=C_{s}(H),
$$

where $S$ is a score function of $L$.

Theorem 1. [5] (De Morgan's laws) Let $H_{1}$ and $H_{2}$ be in HLFE and let $S$ be a score function of L. Then the following laws are valid:

i. $\left(H_{1} \sqcap H_{2}\right)^{c}=H_{1}^{c} \sqcup H_{2}^{c}$,

ii. $\left(H_{1} \sqcup H_{2}\right)^{c}=H_{1}^{c} \sqcap H_{2}^{c}$.

Let $L$ be the set of finite singelton subsets of $[0,1]$. On the lattice $(L, \preceq)$, one complement function can be defined as follows:

$$
C_{S}(H)=\sqcup_{l_{i} \in H}\left\{1-l_{i}\right\}
$$

for every $H \in L$.

Definition 6. [6] A finite automaton is a 5-tuple $\mathcal{A}=\left(Q, X, q_{0}, \varphi, F\right)$ such that

1. $Q$ is a finite nonempty set of states,

2. $X$ is a finite nonempty set of input symbols,

3. $q_{0} \in Q$ is the initial states,

4. $\varphi: Q \times X \rightarrow Q$ is a transition function,

5. $F \subseteq Q$ is the final states.

Definition 7. [10] A partial fuzzy automaton (pfa) is a 5-tuple $\mathcal{A}=(Q, X, \iota, \delta, \tau)$, where

1. $Q$ is a finite nonempty set of states,

2. $X$ is a finite nonempty set of input symbols, 
3. $\iota$ is a fuzzy subset of $Q$, i.e., $\iota: Q \rightarrow[0,1]$, called the initial fuzzy states,

4. $\delta: Q \times X \rightarrow Q$ is a function, call the transition function,

5. $\tau$ is a fuzzy subset of $Q$, i.e., $\tau: Q \rightarrow[0,1]$, called the final fuzzy states.

Definition 8. [6] Let $\mathcal{A}=\left(Q, X, q_{0}, \varphi, F\right)$ be a finite automaton. Then $\mathcal{A}$ computes the language $f_{\mathcal{A}}$ subset of $X^{*}$ by $f_{\mathcal{A}}=\left\{u \in X^{*} \mid \varphi\left(q_{0}, u\right) \in F\right\}$ where $u \in X^{*}$.

The subset $A$ of $X^{*}$ is called recognizable language if there exists a finite automaton $\mathcal{A}$ such that $A=f_{\mathcal{A}}$. The cardinality of $\mathcal{A}$ is defined by $|\mathcal{A}|=|Q|$, where $Q$ is the states of $\mathcal{A}$.

\section{Hesitant L-fuzzy behavior and automaton}

In this section, at first, we present the notion of hesitant L-fuzzy automaton and hesitant L-fuzzy behavior. After that, some of the closure properties for the hesitant L-fuzzy automata such as union and complement are considered, also, the behavior of them are discussed.

Definition 9. A sequential automaton consist of three main structures, the transition structure, the input and output structure. A hesitant L-fuzzy automaton (HLFA) is a quintuple $\mathcal{A}=(Q, X, \iota, \delta, \tau)$, where

1. $Q$ is a finite non-empty set of states,

2. $X$ is a finite set of input symbols,

3. $\iota: Q \rightarrow 2^{L}$ is the hesitant L-fuzzy set on $Q$ of initial states,

4. $\delta: Q \times X \times Q \rightarrow 2^{L}$ is a transition hesitant L-fuzzy set,

5. $\tau: Q \rightarrow 2^{L}$ is the hesitant L-fuzzy set of final states.

For a hesitant L-fuzzy automaton $\mathcal{A}=(Q, X, \iota, \delta, \tau)$, the hesitant L-fuzzy set $\delta$ is extended into a function $\delta^{*}$ : $Q \times X^{*} \times Q \rightarrow 2^{L}$ using the recursion:

$$
\delta^{*}(q, \Lambda, p)=\left\{\begin{array}{ll}
\{0\} & \text { if } q \neq p \\
\{1\} & \text { otherwise }
\end{array},\right.
$$

and

$$
\left.\delta^{*}(q, u a, p)=\sqcup_{r \in Q}\left[\delta^{*}(q, u, r) \sqcap \delta(r, a, p)\right)\right],
$$

where $u \in X^{*}$ and $a \in X$.

Now, we study the concepts of hesitant L-fuzzy behavior and inverse hesitant L-fuzzy behavior recognized by a type of hesitant L-fuzzy automaton. Also, we show that if $\beta_{1}$ and $\beta_{2}$ are regular, then $\beta_{1} \sqcup \beta_{2}$ is regular, too.

Definition 10. Let $\mathcal{A}=(Q, X, \iota, \delta, \tau)$ be an HLFA. Then $\mathcal{A}$ computes the hesitant L-fuzzy behavior (HLFB) $\beta_{\mathcal{A}}: X^{*} \rightarrow 2^{L}$ is defined by

$$
\beta(u)=\sqcup_{q, p \in Q}\left(\iota(q) \sqcap \delta^{*}(q, u, p) \sqcap \tau(p)\right),
$$

where $u \in X^{*}$. 
Definition 11. Let $X$ be a nonempty set of symbols. An HLFS $\beta$ is called regular if there exists an HLFA $\mathcal{A}=(Q, X, \iota, \delta, \tau)$ such that $\beta=\beta_{A}$.

Definition 12. Let $X$ be a nonempty set of symbols, $\beta_{1}: X^{*} \rightarrow 2^{L}$ and $\beta_{2}: X^{*} \rightarrow 2^{L}$ be regular. Then the union of $\beta_{1}$ and $\beta_{2}$, denoted by $\beta_{1} \sqcup \beta_{2}$, is given by $\left(\beta_{1} \sqcup \beta_{2}\right)(u)=\beta_{1}(u) \sqcup \beta_{2}(u)$, for every $u \in X^{*}$.

Theorem 2. Let $\beta_{1}$ and $\beta_{2}$ be regular on $X^{*}$. Then $\beta_{1} \sqcup \beta_{2}$ is regular.

Proof. Let $\beta_{1}$ and $\beta_{2}$ be regular. Then there exist two HLFAs $\mathcal{A}_{1}=\left(Q_{1}, X, \iota_{1}, \delta_{1}, \tau_{1}\right)$ and $\mathcal{A}_{2}=\left(Q_{2}, X, \iota_{2}, \delta_{2}, \tau_{2}\right)$ such that $\beta_{1}=\beta_{\mathcal{A}_{1}}$ and $\beta_{2}=\beta_{\mathcal{A}_{2}}$. Without loss of generality, let $Q_{1} \cap Q_{2}=\emptyset$. Now, consider $\mathcal{A}=(Q, X, \iota, \delta, \tau)$ as follows:

1. $Q=Q_{1} \cup Q_{2}$,

2.

$$
\iota(q)=\left\{\begin{array}{ll}
\iota_{1}(q) & \text { if } q \in Q_{1} \\
\iota_{2}(q) & \text { if } q \in Q_{2}
\end{array},\right.
$$

for every $q \in Q$.

3.

$$
\delta\left(q, a, q^{\prime}\right)= \begin{cases}\delta_{1}\left(q, a, q^{\prime}\right) & \text { if } q, q^{\prime} \in Q_{1} \\ \delta_{2}\left(q, a, q^{\prime}\right) & \text { if } q, q^{\prime} \in Q_{2} \\ \{0\} & \text { otherwise }\end{cases}
$$

for every $q, q^{\prime} \in Q_{1} \cup Q_{2}$.

4.

$$
\tau(q)=\left\{\begin{array}{ll}
\tau_{1}(q) & \text { if } q \in Q_{1} \\
\tau_{2}(q) & \text { if } q \in Q_{2}
\end{array} .\right.
$$

Now, we have $\beta_{\mathcal{A}}(u)=\sqcup_{q, p \in Q} \iota(q) \sqcap \delta(q, u, p) \sqcap \tau(p)$. By definition $\delta$, if $p \in Q_{1}$ and $q \in Q_{2}$, then $\delta(p, a, q)=\{0\}$. So Without loss of generality, let $p_{1}, \ldots, p_{k}, q_{1}, \ldots, q_{k} \in Q_{1}$ and $p_{1}^{\prime}, \ldots, p_{r}^{\prime}, q_{1}^{\prime}, \ldots, q_{r}^{\prime} \in Q_{2}$ such that

$$
\begin{aligned}
\beta_{\mathcal{A}}(u)=\sqcup_{p, q \in Q} \iota(q) \sqcap \delta(q, u, p) \sqcap \tau(p) & =\left(\iota\left(p_{1}\right) \sqcap \delta\left(p_{1}, u, q_{1}\right) \sqcap \tau\left(q_{1}\right)\right) \sqcup\left(\iota\left(p_{2}\right) \sqcap \delta\left(p_{2}, u, q_{2}\right) \sqcap \tau\left(q_{2}\right)\right) \sqcup \\
& \ldots \sqcup\left(\iota\left(p_{k}\right) \sqcap \delta\left(p_{k}, u, q_{k}\right) \sqcap \tau\left(q_{k}\right)\right) \sqcup\left(\iota\left(p_{1}^{\prime}\right) \sqcap \delta\left(p_{1}^{\prime}, u, q_{1}^{\prime}\right) \sqcap \tau\left(q_{1}^{\prime}\right)\right) \sqcup \\
& \ldots \sqcup\left(\iota\left(p_{r}^{\prime}\right) \sqcap \delta\left(p_{r}^{\prime}, u, q_{r}^{\prime}\right) \sqcap \tau\left(q_{r}^{\prime}\right)\right) \\
& =\beta_{\mathcal{A}_{1}}(u) \sqcup \beta_{\mathcal{A}_{2}}(u) .
\end{aligned}
$$

Example 1. Let the bounded lattice $L$ as Figure 1. Let two HLFAs $\mathcal{A}_{1}=\left(Q_{1}, X, \iota_{1}, \delta_{1}, \tau_{1}\right)$ and $\mathcal{A}_{2}=\left(Q_{2}, X, \iota_{2}\right.$, $\left.\delta_{2}, \tau_{2}\right)$ such that $Q_{1}=\left\{q_{1}, q_{2}, q_{3}\right\}, X=\{a, b\}, \iota_{1}\left(q_{1}\right)=\{\alpha, \gamma\}, \delta_{1}\left(q_{1}, a, q_{2}\right)=\{\beta, \gamma\}, \delta_{1}\left(q_{1}, b, q_{2}\right)=\{\alpha\}, \delta_{1}\left(q_{1}\right.$, $\left.a, q_{3}\right)=\{\alpha, \gamma\}, \tau_{1}\left(q_{2}\right)=\{\gamma\}, \tau_{1}\left(q_{3}\right)=\{\beta\}$ and $Q_{2}=\left\{p_{1}, p_{2}, p_{3}\right\}, \iota_{2}\left(p_{1}\right)=\{\eta\}, \delta_{2}\left(p_{1}, a, p_{2}\right)=\{\beta, \xi\}, \delta_{2}\left(p_{1}, a\right.$, 


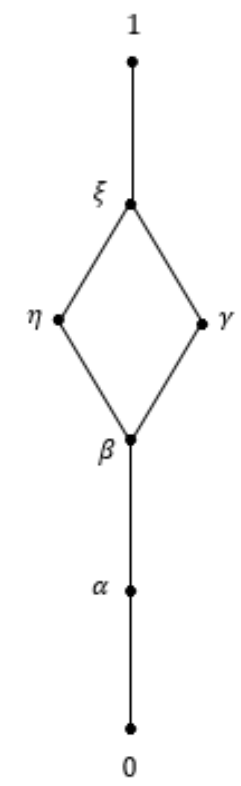

Figure 1: The bounded lattice $L$ of Example 1

$\left.p_{3}\right)=\{\beta, \eta\}, \delta_{2}\left(p_{2}, b, p_{3}\right)=\{\alpha\}, \tau_{2}\left(p_{2}\right)=\{\eta\}, \tau_{2}\left(p_{3}\right)=\{\xi\}$. Now, consider $\mathcal{A}=(Q, X, \iota, \delta, \tau)$ such that $Q=\left\{q_{1}, q_{2}, q_{3}, p_{1}, p_{2}, p_{3}\right\}, X=\{a, b\}$ and

$$
\begin{array}{rlrl}
\iota\left(q_{1}\right) & =\{\alpha, \gamma\}, & \iota\left(p_{1}\right)=\{\eta\} \\
\delta\left(q_{1}, a, q_{2}\right)=\{\beta, \gamma\}, & \delta\left(q_{1}, b, q_{2}\right)=\{\alpha\}, \\
\delta\left(q_{1}, a, q_{3}\right)=\{\alpha, \gamma\}, & \delta\left(p_{1}, a, p_{2}\right)=\{\beta, \xi\}, \\
\delta\left(p_{1}, a, p_{3}\right)=\{\beta, \eta\}, & \delta\left(p_{2}, b, p_{3}\right)=\{\alpha\},
\end{array}
$$

and

$$
\begin{array}{ll}
\tau\left(q_{2}\right)=\{\gamma\}, & \tau\left(q_{3}\right)=\{\beta\} \\
\tau\left(p_{2}\right)=\{\eta\}, & \tau\left(p_{3}\right)=\{\xi\} .
\end{array}
$$

Therefore,

$$
\begin{aligned}
\beta_{\mathcal{A}}(a)= & \sqcup_{p, q \in Q} \iota(p) \sqcap \delta(p, a, q) \sqcap \tau(q) \\
= & \left(\iota\left(q_{1}\right) \sqcap \delta\left(q_{1}, a, q_{2}\right) \sqcap \tau\left(q_{2}\right)\right) \sqcup\left(\iota\left(q_{1}\right) \sqcap \delta\left(q_{1}, a, q_{3}\right) \sqcap \tau\left(q_{3}\right)\right) \\
& \sqcup\left(\iota\left(p_{1}\right) \sqcap \delta\left(p_{1}, a, p_{2}\right) \sqcap \tau\left(p_{2}\right)\right) \sqcup\left(\iota\left(p_{1}\right) \sqcap \delta\left(p_{1}, a, p_{3}\right) \sqcap \tau\left(p_{3}\right)\right) \\
= & (\{\alpha, \gamma\} \sqcap\{\beta, \gamma\} \sqcap\{\gamma\}) \sqcup(\{\alpha, \gamma\} \sqcap\{\alpha, \gamma\} \sqcap\{\beta\}) \\
& \quad \sqcup(\{\eta\} \sqcap\{\beta, \xi\} \sqcap\{\eta\}) \sqcup(\{\eta\} \sqcap\{\beta, \eta\} \sqcap\{\xi\}) \\
= & \{\alpha, \beta, \gamma\} \sqcup\{\alpha, \beta\} \sqcup\{\beta, \eta\} \sqcup\{\beta, \eta\} \\
= & \{\beta, \eta, \gamma, \xi\} .
\end{aligned}
$$


Also, we have $\beta_{\mathcal{A}_{1}}(a)$ and $\beta_{\mathcal{A}_{2}}(a)$ as follows:

$$
\begin{aligned}
\beta_{\mathcal{A}_{1}}(a) & =\sqcup_{p, q \in Q_{1}} \iota_{1}(p) \sqcap \delta_{1}(p, a, q) \sqcap \tau_{1}(q) \\
& =\left(\iota_{1}\left(q_{1}\right) \sqcap \delta_{1}\left(q_{1}, a, q_{2}\right) \sqcap \tau_{1}\left(q_{2}\right)\right) \sqcup\left(\iota_{1}\left(q_{1}\right) \sqcap \delta_{1}\left(q_{1}, a, q_{3}\right) \sqcap \tau_{1}\left(q_{3}\right)\right) \\
& =(\{\alpha, \gamma\} \sqcap\{\beta, \gamma\} \sqcap\{\gamma\}) \sqcup(\{\alpha, \gamma\} \sqcap\{\alpha, \gamma\} \sqcap\{\beta\}) \\
& =\{\alpha, \beta, \gamma\} \sqcup\{\alpha, \beta\} \\
& =\{\alpha, \beta, \gamma\},
\end{aligned}
$$

and

$$
\begin{aligned}
\beta_{\mathcal{A}_{2}}(a) & =\sqcup_{p, q \in Q_{2}} \iota_{2}(p) \sqcap \delta_{2}(p, a, q) \sqcap \tau_{2}(q) \\
& =\left(\iota_{2}\left(p_{1}\right) \sqcap \delta_{2}\left(p_{1}, a, p_{2}\right) \sqcap \tau_{2}\left(p_{2}\right)\right) \sqcup\left(\iota_{2}\left(p_{1}\right) \sqcap \delta_{2}\left(p_{1}, a, p_{3}\right) \sqcap \tau_{2}\left(p_{3}\right)\right) \\
& =(\{\eta\} \sqcap\{\beta, \xi\} \sqcap\{\eta\}) \sqcup(\{\eta\} \sqcap\{\beta, \eta\} \sqcap\{\xi\}) \\
& =\{\beta, \eta\} \sqcap\{\beta, \eta\} \\
& =\{\beta, \eta\} .
\end{aligned}
$$

So, we have

$$
\beta_{\mathcal{A}_{1}}(a) \sqcup \beta_{\mathcal{A}_{2}}(a)=\{\alpha, \beta, \gamma\} \sqcup\{\beta, \eta\}=\{\beta, \eta, \gamma, \xi\} .
$$

Hence, clearly, $\beta_{\mathcal{A}_{1}}(a) \sqcup \beta_{\mathcal{A}_{2}}(a)=\beta_{\mathcal{A}}(a)$

Corollary 1. Let $\left\{H_{i}\right\}_{i \in I}$ be a finite family of regular HLFB, for every $i \in I$. Then $\sqcup_{i \in I} H_{i}$ is regular.

Definition 13. Let $\mathcal{A}=(Q, X, \iota, \delta, \tau)$ be an HLFA. Then the inverse hesitant L-fuzzy behavior $\bar{\beta}: X^{*} \rightarrow 2^{L}$ is defined by

$$
\bar{\beta}(u)=\sqcap_{q, p \in Q}\left(\iota(q) \sqcup \delta^{*}(q, u, p) \sqcup \tau(p)\right),
$$

where $u \in X^{*}$.

Theorem 3. Let $\mathcal{A}=(Q, X, \iota, \delta, \tau)$ be an HLFA. Then there exists an $H L F A \mathcal{A}^{c}$ such that $\bar{\beta}_{\mathcal{A}^{c}}(u)=C_{S}\left(\beta_{\mathcal{A}}(u)\right)$, for every $u \in X^{*}$.

Proof. Let $\mathcal{A}_{c}=\left(Q, X, \iota^{c}, \delta^{c}, \tau^{c}\right)$, where $\iota^{c}=C_{S}(\iota), \delta^{c}(p, a, q)=C_{S}(\delta)$ and $\tau^{c}=C_{S}(\tau)$. By considering Theorem 1, we have

$$
\delta^{c}(p, \Lambda, q)=\left\{\begin{array}{ll}
\{1\} & \text { if } p \neq q \\
\{0\} & \text { if } p=q
\end{array},\right.
$$

and

$$
\begin{aligned}
C_{S}(\delta)=\delta^{c}(p, u a, q) & =(\delta(p, u a, q))^{c}=\left(\sqcup_{q^{\prime} \in Q} \delta\left(p, u, q^{\prime}\right) \sqcap \delta\left(q^{\prime}, a, q\right)\right)^{c} \\
& =\sqcap_{q^{\prime} \in Q} \delta^{c}\left(p, u, q^{\prime}\right) \sqcup \delta^{c}\left(q^{\prime}, a, q\right),
\end{aligned}
$$


where $u \in X^{*}, a \in X$ and $p, q \in Q$. Now, by considering Definition 5 and Theorem 1, we have

$$
\begin{aligned}
\bar{\beta}_{\mathcal{A}^{c}}(u) & =\sqcap_{p, q \in Q}\left(\iota^{c}(p) \sqcup \delta^{c *}(p, u, q) \sqcup \tau^{c}(q)\right) \\
& =\sqcap_{p, q \in Q}\left(\iota(p) \sqcap \delta^{*}(p, u, q) \sqcap \tau(q)\right)^{c} \\
& =\left(\sqcup_{p, q \in Q} \iota(p) \sqcap \delta^{*}(p, u, q) \sqcap \tau(q)\right)^{c} \\
& =\left(\beta_{\mathcal{A}}(u)\right)^{c} \\
& =C_{S}\left(\beta_{\mathcal{A}}(u)\right) .
\end{aligned}
$$

Example 2. Let $\mathcal{A}=(Q, X, \iota, \delta, \tau)$ be an HLFA, where $Q=\left\{q_{1}, q_{2}, q_{3}\right\}, \iota\left(q_{1}\right)=\{0.5,0.7\}, X=\{a, b\}$,

$$
\begin{array}{ll}
\delta\left(q_{1}, a, q_{1}\right)=\{0.3,0.4\}, & \delta\left(q_{1}, b, q_{2}\right)=\{0.2,0.4\}, \\
\delta\left(q_{1}, a, q_{3}\right)=\{0.6,0.7\}, & \delta\left(q_{2}, a, q_{1}\right)=\{0.7\}, \\
\delta\left(q_{3}, b, q_{2}\right)=\{0.1,0.4\}, &
\end{array}
$$

and $\tau\left(q_{2}\right)=\{0.6\}$. By considering $C_{S}(H)=\sqcup_{l_{i} \in H}\left\{1-l_{i}\right\}$, for every $H \in 2^{L}$, we have $\mathcal{A}^{c}$ as follows: $\mathcal{A}^{c}=\left(Q, X, \iota^{c}, \delta^{c}, \tau^{c}\right)$, where $\iota^{c}\left(q_{1}\right)=\{0.3,0.5\}$,

$$
\begin{aligned}
& \delta^{c}\left(q_{1}, a, q_{1}\right)=\{0.6,0.7\}, \quad \delta^{c}\left(q_{1}, b, q_{2}\right)=\{0.6,0.8\} \\
& \delta^{c}\left(q_{1}, a, q_{3}\right)=\{0.3,0.4\}, \quad \delta^{c}\left(q_{2}, a, q_{1}\right)=\{0.3\} \\
& \delta^{c}\left(q_{3}, b, q_{2}\right)=\{0.6,0.9\}, \quad \tau^{c}\left(q_{2}\right)=\{0.4\} .
\end{aligned}
$$

So, we have

$$
\begin{aligned}
\bar{\beta}_{\mathcal{A}^{c}}(a b) & =\sqcap_{p, q \in Q}\left(\iota^{c}(p) \sqcup \delta^{c}(p, a b, q) \sqcup \tau^{c}(q)\right) \\
& =\iota^{c}\left(q_{1}\right) \sqcup \delta^{c}\left(q_{1}, a b, q_{2}\right) \sqcup \tau^{c}\left(q_{2}\right) .
\end{aligned}
$$

By considering (1), we have

$$
\begin{aligned}
\delta^{c}\left(q_{1}, a b, q_{2}\right) & =\left(\delta^{c}\left(q_{1}, a, q_{1}\right) \sqcup \delta^{c}\left(q_{1}, b, q_{2}\right)\right) \sqcap\left(\delta^{c}\left(q_{1}, a, q_{3}\right) \sqcup \delta^{c}\left(q_{3}, b, q_{2}\right)\right) \\
& =(\{0.6,0.7\} \sqcup\{0.6,0.8\}) \sqcap(\{0.3,0.4\} \sqcup\{0.6,0.9\}) \\
& =\{0.6,0.7,0.8\} \sqcap\{0.6,0.9\} \\
& =\{0.6,0.7,0.8\} .
\end{aligned}
$$

So,

$$
\begin{aligned}
\bar{\beta}_{\mathcal{A}^{c}}(a b) & =\iota^{c}\left(q_{1}\right) \sqcup \delta^{c}\left(q_{1}, a b, q_{2}\right) \sqcup \tau^{c}\left(q_{2}\right) \\
& =\{0.3,0.5\} \sqcup\{0.6,0.7,0.8\} \sqcup\{0.4\} \\
& =\{0.6,0.7,0.8\} .
\end{aligned}
$$


In the other hand

$$
\begin{aligned}
\delta\left(q_{1}, a b, q_{2}\right) & =\left(\delta\left(q_{1}, a, q_{1}\right) \sqcap \delta\left(q_{1}, b, q_{2}\right)\right) \sqcup\left(\delta\left(q_{1}, a, q_{3}\right) \sqcap \delta\left(q_{3}, b, q_{2}\right)\right) \\
& =(\{0.3,0.4\} \sqcap\{0.2,0,4\}) \sqcup(\{0.6,0.7\} \sqcap\{0.1,0.4\}) \\
& =\{0.2,0.3,0.4\} \sqcup\{0.1,0.4\} \\
& =\{0.2,0.3,0.4\}
\end{aligned}
$$

Then we have $\beta_{\mathcal{A}}(a b)$ as follow:

$$
\begin{aligned}
\beta_{\mathcal{A}}(a b) & =\iota\left(q_{1}\right) \sqcap \delta\left(q_{1}, a b, q_{2}\right) \sqcap \tau\left(q_{2}\right) \\
& =\{0.5,0.7\} \sqcap\{0.2,0.3,0.4\} \sqcap\{0.6\} \\
& =\{0.2,0.3,0.4\} .
\end{aligned}
$$

Hence, $C_{S}\left(\beta_{\mathcal{A}}(a b)\right)=\{0.6,0.7,0.8\}$.

Now, we show that if $\beta$ is an HLFB recognized by an incomplete HLFA $\mathcal{A}$, then there exists a complete HLFA $\mathcal{A}_{c}$ of $\mathcal{A}$ such that $\beta_{\mathcal{A}_{c}}=\beta_{\mathcal{A}}$. We also prove that if $A$ is regular, then there exists a complete accessible HLFA $\mathcal{A}_{c a}$ such that $\beta_{\mathcal{A}_{c a}}=A$.

Definition 14. Let $\mathcal{A}=(Q, X, \iota, \delta, \tau)$ be an HLFA. Then $\mathcal{A}$ is called complete if for every $q \in Q$ and $a \in X$, there exist $p \in Q$ and $0 \neq \alpha \in L$ such that $\alpha \in \delta(q, a, p)$.

Theorem 4. Let $\mathcal{A}=(Q, X, \iota, \delta, \tau)$ be an incomplete HLFA. Then there exists a complete HLFA $\mathcal{A}_{c}$ such that $\beta_{\mathcal{A}}=\beta_{\mathcal{A}_{c}}$.

Proof. Let $\mathcal{A}=(Q, X, \iota, \delta, \tau)$ be an incomplete HLFA. Let $\mathcal{A}_{c}=\left(Q_{c}, X, \iota_{c}, \delta_{c}, \tau_{c}\right)$ as follows: $Q_{c}=Q \cup\{t\}$, where $t \notin Q$ and define

$$
\begin{gathered}
\iota_{c}: Q_{c} \rightarrow 2^{L} \\
\iota_{c}(q)=\left\{\begin{array}{ll}
\iota(q) & \text { if } q \in Q \\
\{0\} & \text { if } q=t
\end{array},\right. \\
\tau_{c}: Q_{c} \rightarrow 2^{L} \\
\tau_{c}(q)=\left\{\begin{array}{ll}
\tau(q) & \text { if } q \in Q \\
\{0\} & \text { if } q=t
\end{array},\right.
\end{gathered}
$$

and $\delta_{c}: Q_{c} \times X \times Q_{c} \rightarrow 2^{L}$, for every $p, q \in Q$ and $a \in X$ consider $\delta_{c}(p, a, q)=\delta(p, a, q)$, also consider

$$
\delta_{c}(p, a, t)=\left\{\begin{array}{ll}
\{\alpha\} & \text { if } \sqcup_{q \in Q} \delta(p, a, q)=\{0\} \\
\{0\} & \text { otherwise }
\end{array},\right.
$$

and

$$
\delta_{c}(t, a, p)= \begin{cases}\{\alpha\} & \text { if } p=t \\ \{0\} & \text { otherwise }\end{cases}
$$


where $a \in X$. Clearly, $\mathcal{A}_{c}$ is a complete HLFA. By considering the definition of $\mathcal{A}_{c}$ we have

$$
\begin{aligned}
& \beta_{\mathcal{A}}(x)=\sqcup_{p, q \in Q} \iota(p) \sqcap \delta(p, x, q) \sqcap \tau(q) \\
& =\sqcup_{p, q \in Q} \iota_{c}(p) \sqcap \delta_{c}(p, x, q) \sqcap \tau_{c}(q) \\
& =\left(\sqcup_{p, q \in Q} \iota_{c}(p) \sqcap \delta_{c}(p, x, q) \sqcap \tau_{c}(q)\right) \sqcup\left(\sqcup_{q \in Q} \iota_{c}(t) \sqcap \delta_{c}(t, x, q) \sqcap \tau_{c}(q)\right) \sqcup\left(\sqcup_{p \in Q} \iota_{c}(p) \sqcap \delta_{c}(p, x, t) \sqcap \tau_{c}(t)\right)
\end{aligned}
$$

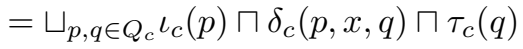

$$
\begin{aligned}
& =\beta_{\mathcal{A}_{c}}(x) \text {. }
\end{aligned}
$$

Definition 15. Let $\mathcal{A}=(Q, X, \iota, \delta, \tau)$ be an HLFA. Let $S=\left\{q \in Q \mid \sqcup\left\{\sqcup\left\{\iota(p) \sqcap \delta^{*}(p, x, q) \mid p \in Q\right\} \mid x \in X^{*}\right\} \neq\right.$ $\{0\}$ \}. Then we say that $\mathcal{A}$ is accessible if $S=Q$.

Theorem 5. Let $\mathcal{A}=(Q, X, \iota, \delta, \tau)$ be an HLFA. Then there exists a complete accessible HLFA $\mathcal{A}_{c a}$ such that $\beta_{\mathcal{A}}(x)=\beta_{\mathcal{A}_{c a}}(x)$, where $\beta_{\mathcal{A}}(x) \neq\{0\}$, for some $x \in X^{*}$.

Proof. By Theorem 4, there exists a complete HLFA $\mathcal{A}_{c}=\left(Q_{c}, X, \iota_{c}, \delta_{c}, \tau_{c}\right)$ such that $\beta_{\mathcal{A}}=\beta_{\mathcal{A}_{c}}$. Now, consider HLFA $\mathcal{A}_{c a}$ as follows: $\mathcal{A}_{c a}=\left(Q_{c a}, X, \iota_{c a}, \delta_{c a}, \tau_{c a}\right)$ such that $Q_{c a}=\left\{q \in Q_{c} \mid \sqcup\left\{\sqcup\left\{\iota_{c}(p) \sqcap \delta_{c}^{*}(p, x, q) \mid p \in\right.\right.\right.$ $\left.\left.Q\} \mid x \in X^{*}\right\} \neq\{0\}\right\}$ and $\delta_{c a}$ is the restriction of $\delta_{c}$ over $Q_{c a} \times X \times Q_{c a}, \iota_{c a}$ is the restriction of $\iota_{c}$ over $Q_{c a}$ and $\tau_{c a}$ is the restriction of $\tau_{c}$ over $Q_{c a}$. Now, let $\beta_{\mathcal{A}_{c}}(x)=\sqcup_{p, q \in Q} \iota_{c}(p) \sqcap \delta_{c}^{*}(p, x, q) \sqcap \tau_{c}(q) \neq\{0\}$. Without loss of generality let $p_{1}, p_{2}, \ldots, p_{k}, q_{1}, q_{2}, \ldots, q_{k} \in Q_{c}$, not necessarily distinct, such that

$$
\begin{aligned}
& \sqcup_{p, q \in Q} \iota_{c}(p) \sqcap \delta_{c}^{*}(p, x, q) \sqcap \tau_{c}(q)=\left(\iota_{c}\left(p_{1}\right) \sqcap \delta_{c}^{*}\left(p_{1}, x, q_{1}\right) \sqcap \tau_{c}\left(q_{1}\right)\right) \\
& \quad \sqcup\left(\iota_{c}\left(p_{2}\right) \sqcap \delta_{c}^{*}\left(p_{2}, x, q_{2}\right) \sqcap \tau_{c}\left(q_{2}\right)\right) \sqcup \ldots \sqcup\left(\iota_{c}\left(p_{k}\right) \sqcap \delta_{c}^{*}\left(p_{k}, x, q_{k}\right) \sqcap \tau_{c}\left(q_{k}\right)\right) .
\end{aligned}
$$

If $\iota_{c}\left(p_{i}\right) \sqcap \delta_{c}^{*}\left(p_{i}, x, q_{i}\right)=\{0\}$, for $i \in\{1, \ldots, k\}$, then $\iota_{c}\left(p_{i}\right) \sqcap \delta_{c}^{*}\left(p_{i}, x, q_{i}\right) \sqcap \tau_{c}\left(q_{i}\right)=\{0\}$ and $\{0\} \sqcup H=H$, where $H \in 2^{L}$. Without loss of generality, let $\iota_{c}\left(p_{i}\right) \sqcap \delta_{c}^{*}\left(p_{i}, x, q_{i}\right)=\{0\}$, for $1 \leq i \leq r$ and $\iota_{c}\left(p_{j}\right) \sqcap \delta_{c}^{*}\left(p_{j}, x, q_{j}\right) \neq\{0\}$, for $r<j \leq k$. By using $\beta_{\mathcal{A}_{c}}(x) \neq\{0\}$ we have

$$
\begin{aligned}
\left(\iota_{c}\left(p_{1}\right) \sqcap \delta_{c}^{*}\left(p_{1}, x, q_{1}\right) \sqcap \tau_{c}\left(q_{1}\right)\right) & \sqcup\left(\iota_{c}\left(p_{2}\right) \sqcap \delta_{c}^{*}\left(p_{2}, x, q_{2}\right) \sqcap \tau_{c}\left(q_{2}\right)\right) \\
& \sqcup \ldots \sqcup\left(\iota_{c}\left(p_{k}\right) \sqcap \delta_{c}^{*}\left(p_{k}, x, q_{k}\right) \sqcap \tau_{c}\left(q_{k}\right)\right) \\
= & \left(\iota_{c}\left(p_{r+1}\right) \sqcap \delta_{c}^{*}\left(p_{r+1}, x, q_{r+1}\right) \sqcap \tau_{c}\left(q_{r+1}\right)\right) \sqcup\left(\iota_{c}\left(p_{r+2}\right) \sqcap \delta_{c}^{*}\left(p_{r+2}, x, q_{r+2}\right) \sqcap \tau_{c}\left(q_{r+2}\right)\right) \\
& \sqcup \ldots \sqcup\left(\iota_{c}\left(p_{k}\right) \sqcap \delta_{c}^{*}\left(p_{k}, x, q_{k}\right) \sqcap \tau_{c}\left(q_{k}\right)\right) \\
= & \left(\iota_{c a}\left(p_{r+1}\right) \sqcap \delta_{c}^{*}\left(p_{r+1}, x, q_{r+1}\right) \sqcap \tau_{c a}\left(q_{r+1}\right)\right) \sqcup \ldots \sqcup\left(\iota_{c a}\left(p_{k}\right) \sqcap \delta_{c}^{*}\left(p_{k}, x, q_{k}\right) \sqcap \tau_{c a}\left(q_{k}\right)\right) \\
= & \beta_{\mathcal{A}_{c a}}(x) .
\end{aligned}
$$

Hence, the claim holds.

\section{Equivalence classes and properties of hesitant L-fuzzy behavior}

In this section, by considering the hesitant L-fuzzy behavior $\beta$ we give a congruence relation, and by using the equivalence classes we present an HLFA recognized $\beta$. 
Definition 16. Let $\mathcal{A}=(Q, X, \iota, \delta, \tau)$ be an HLFA. Let $Q=\left\{q_{1}, q_{2}, \ldots, q_{n}\right\}$ and $\iota\left(q_{i}\right)=l_{i}$, where $l_{i} \subseteq L$ and $1 \leq i \leq n$. Define $p^{-1} \tau: X^{*} \rightarrow 2^{L}$ by $\left(p^{-1} \tau\right)(x)=\sqcup_{q \in Q} \iota(p) \sqcap \delta^{*}(p, x, q) \sqcap \tau(q)$, where $p \in Q$ and $x \in X^{*}$. Also, consider

$$
\left(p_{1}, p_{2}, \ldots, p_{n}\right)^{-1} \tau(x)=\sqcup_{i=1, q \in Q}^{n} l_{i} \sqcap \delta^{*}\left(p_{i}, x, q\right) \sqcap \tau(q)=\sqcup_{i=1}^{n}\left(p_{i}^{-1} \tau\right)(x),
$$

for every $x \in X^{*}$. Now, let $\mathcal{M}=\left\{\left(p_{1}, p_{2}, \ldots, p_{n}\right)^{-1} \tau \mid p_{i} \in Q, 1 \leq i \leq n\right\} . \mathcal{M}$ is a finite HLFS, since $Q$ is a finite set. Let $\beta$ be a hesitant L-fuzzy set on $X^{*}$. Then for every $u \in X^{*}$, define

$$
\begin{aligned}
& u^{-1} \beta: X^{*} \rightarrow 2^{L} \\
& u^{-1} \beta(v)=\beta(u v)
\end{aligned}
$$

for every $v \in X^{*}$.

Theorem 6. Let $\beta$ be an HLFB. Then $\mathcal{N}=\left\{u^{-1} \beta \mid u \in X^{*}\right\}$ is a finite hesitant L-fuzzy set.

Proof. Let $\beta$ be an HLFB. Then

$$
\begin{aligned}
\left(u^{-1} \beta\right)(x)= & \beta(u x) \\
= & \sqcup_{q, p \in Q}\left(\iota(q) \sqcap \delta^{*}(q, u x, p) \sqcap \tau(p)\right) \\
= & \sqcup_{p \in Q}\left(\iota\left(q_{1}\right) \sqcap \delta^{*}\left(q_{1}, u x, p\right) \sqcap \tau(p)\right) \sqcup \sqcup_{p \in Q}\left(\iota\left(q_{2}\right) \sqcap \delta^{*}\left(q_{2}, u x, p\right) \sqcap \tau(p)\right) \\
& \sqcup \ldots \sqcup \sqcup_{p \in Q}\left(\iota\left(q_{n}\right) \sqcap \delta^{*}\left(q_{n}, u x, p\right) \sqcap \tau(p)\right) \\
= & \sqcup_{p \in Q}\left(l_{1} \sqcap \delta^{*}\left(q_{1}, u x, p\right) \sqcap \tau(p)\right) \sqcup \sqcup_{p \in Q}\left(l_{2} \sqcap \delta^{*}\left(q_{2}, u x, p\right) \sqcap \tau(p)\right) \\
& \sqcup \ldots \sqcup \sqcup_{p \in Q}\left(l_{n} \sqcap \delta^{*}\left(q_{n}, u x, p\right) \sqcap \tau(p)\right) \\
= & \left(q_{1}, q_{2}, \ldots, q_{n}\right)^{-1} \tau(u x),
\end{aligned}
$$

where $u, x \in X^{*}$. Therefore, $\left(u^{-1} \beta\right)(x)=\left(q_{1}, q_{2}, \ldots, q_{n}\right)^{-1} \tau(u x) \in \mathcal{M}$. So,

$$
\mathcal{N}=\left\{u^{-1} \beta \mid u \in X^{*}\right\}=\left\{\left(p_{1}, p_{2}, \ldots, p_{n}\right)^{-1} \tau \mid p_{i} \in Q, 1 \leq i \leq n\right\}=\mathcal{M} .
$$

Since $\mathcal{M}$ is a finite HLFS, then $\mathcal{N}$ is a finite HLFS, too.

Theorem 7. Let $\beta$ be an HLFS on $X^{*}$. Then the following are equivalent:

1. $\beta$ is an $H L F B$,

2. $\mathcal{K}=\left\{(u, v) \in X^{*} \times X^{*} \mid u^{-1} \beta=v^{-1} \beta\right\}$ is a right congruence of finite index,

3. $\mathcal{R}=\left\{(u, v) \in X^{*} \times X^{*} \mid \beta(x u y)=\beta(x v y)\right.$ for every $\left.x, y \in X^{*}\right\}$ is a right congruence of finite index.

Proof. 1. $\Rightarrow 2$. It is obvious that $\mathcal{K}$ is a equivalence relation on $X^{*}$. By considering Theorem $6, \mathcal{N}$ is a finite set, so we can say that $\mathcal{K}$ is a finite set. Let $u \mathcal{K} v$. Then $u^{-1} \beta=v^{-1} \beta$. Therefore, $u^{-1} \beta(w)=v^{-1} \beta(w)$, for every $w \in X^{*}$. Then $\beta(u w)=\beta(v w)$, for every $w \in X^{*}$. Now, by considering $w=x y$ we have $\beta(u x y)=\beta(v x y)$, for every $y \in X^{*}$. So, $(u x)^{-1} \beta=(v x)^{-1} \beta$. Hence, $\mathcal{K}$ is a right congruence of finite index. 
2. $\Rightarrow 1$. Let $\mathcal{A}=(Q, X, \iota, \delta, \tau)$ be an HLFA, where $Q$ is the set of all $\mathcal{K}$-equivalence classes, and $\iota, \delta$ and $\tau$ are as follows:

$$
\begin{gathered}
\iota: Q \rightarrow 2^{L} \\
\iota([x])=\left\{\begin{array}{ll}
\{1\} & \text { if }[x]=[\Lambda] \\
\{0\} & \text { if }[x] \neq[\Lambda]
\end{array},\right. \\
\delta: Q \times X \times Q \rightarrow 2^{L} \\
\delta([x], a,[y])=\left\{\begin{array}{ll}
\{1\} & \text { if }[y]=[x a] \\
\{0\} & \text { otherwise }
\end{array},\right.
\end{gathered}
$$

and

$$
\begin{aligned}
& \tau: Q \rightarrow 2^{L} \\
& \tau([x])=\beta(x),
\end{aligned}
$$

for every $[x],[y] \in Q$ and $a \in X$. Now, we show that $\delta$ is well-defined. Let $[u]=[v]$ and $[x]=[y]$. Then $u \mathcal{K} v$ and $x \mathcal{K} y$. Since $\mathcal{K}$ is right congruence, so $u a \mathcal{K} v a$. Then $[u a]=[x]$, implies that $[v a]=[y]$. Therefore, $\delta([u], a,[x])=\delta([v], a,[y])$. Hence, $\delta$ is well-defined.

Now, let $[x]=[y]$. Then $x \mathcal{K} y$ implies that $x^{-1} \beta=y^{-1} \beta$. Therefore, $x^{-1} \beta(\Lambda)=y^{-1} \beta(\Lambda)$ implies that $\beta(x)=\beta(y)$. So, $\tau([x])=\tau([y])$. Let $x \in X^{*}$. Then

$$
\begin{aligned}
\beta_{\mathcal{A}}(x)=\sqcup_{p, q \in Q} \iota(p) \sqcap \delta^{*}(p, x, q) \sqcap \tau(q) & =\sqcup_{[x],[y]} \iota([x]) \sqcap \delta^{*}([x], x,[y]) \sqcap \tau([y]) \\
& =\iota([\Lambda]) \sqcap \delta^{*}([\Lambda], x,[x]) \sqcap \tau([x]) \\
& =\{1\} \sqcap\{1\} \sqcap \tau([x]) \\
& =\tau([x]) \\
& =\beta(x) .
\end{aligned}
$$

Hence, $\beta$ is a regular HLFB.

1. $\Rightarrow 3$. Let $\beta$ be regular. Clearly, $\mathcal{R}$ is a finite set. Now, let $u \mathcal{R} v$. Then $\beta(x u w)=\beta(x v w)$, for every $x, w \in X^{*}$. So, $\beta($ xuay $)=\beta(x v a y)$, where $w=a y$ and $x, y \in X^{*}$. Then $u a \mathcal{R} v a$. Therefore, $\mathcal{R}$ is a right congruence. 3 . $\Rightarrow 1$. The proof of this part is similar to $2 \Rightarrow 1$.

\section{Minimization of hesitant L-fuzzy automaton}

In this section, at first, we present the notion of hesitant L-fuzzy language. Also, we show that for any hesitant L-fuzzy language $A$, there exists a minimal complete deterministic hesitant L-fuzzy automaton recognizing $A$.

In this section, let $L$ be a lattice such that for every $\alpha \neq 0$ and $\beta \neq\{0\}$ belong $L$, we have been $\alpha \wedge \beta \neq 0$.

Definition 17. Let $\mathcal{A}=(Q, X, \iota, \delta, \tau)$ be an HLFA. Then the hesitant L-fuzzy language recognized by $\mathcal{A}$ is a subset of $X^{*}$ by $\mathcal{L}_{\mathcal{A}}=\left\{x \in X^{*} \mid \iota(p) \sqcap \delta^{*}(p, x, q) \sqcap \tau(q) \neq\{0\}\right.$, for some $\left.p, q \in Q\right\}=\left\{x \in X^{*} \mid \beta(x) \neq\{0\}\right\}$. 
The subset $A$ of $X^{*}$ is called recognizable hesitant L-fuzzy language if there exists an HLFA $\mathcal{A}$ such that $A=\mathcal{L}_{\mathcal{A}}$.

Definition 18. Let $\mathcal{A}=(Q, X, \iota, \delta, \tau)$ be an HLFA. Then we say $\mathcal{A}$ is deterministic if the following holds:

1. There exists a unique $q_{0} \in Q$ such that $\iota\left(q_{0}\right)=\{1\}$.

2. For every $q \in Q$ and $a \in X$, there exists at most one $q^{\prime} \in Q$ such that $\delta\left(q, a, q^{\prime}\right) \neq\{0\}$.

Corollary 2. Let $\mathcal{A}=(Q, X, \iota, \delta, \tau)$ be an HLFA and $\mathcal{A}$ be a complete deterministic HLFA. Then for every $p \in Q$ and $a \in X$, there exists exactly one state $q \in Q$ such that $\delta(q, a, p) \neq\{0\}$.

Theorem 8. Let $\mathcal{A}=(Q, X, \iota, \delta, \tau)$ be an HLFA and $\iota(q) \neq\{0\}$, for some $q \in Q$. Then there exists a deterministic HLFA $\mathcal{A}_{d}$ such that $\mathcal{L}_{\mathcal{A}}=\mathcal{L}_{\mathcal{A}_{d}}$.

Proof. Let $Q_{x}=\left\{q^{\prime} \in Q \mid \iota(q) \sqcap \delta^{*}\left(q, x, q^{\prime}\right) \neq\{0\}\right.$, for some $\left.q \in Q\right\}$, for every $x \in X^{*}$. Then $Q_{\Lambda}=\left\{q^{\prime} \in\right.$ $Q \mid \iota(q) \neq\{0\}\}$. Let $Q_{d}=\left\{Q_{x} \mid x \in X^{*}\right\}$. Define $\delta_{d}: Q_{d} \times X \times Q_{d} \rightarrow 2^{L}$ by

$$
\delta_{d}\left(Q_{y}, a, Q_{x}\right)= \begin{cases}\{\alpha\} & \text { if } Q_{y a}=Q_{x} \\ \{0\} & \text { otherwise }\end{cases}
$$

let $\iota_{d}: Q_{d} \rightarrow 2^{L}$ by $\iota_{d}\left(Q_{\Lambda}\right)=\{\alpha\}$ and $\iota_{d}\left(Q^{\prime}\right)=\{0\}$ for every $Q^{\prime} \neq Q_{\Lambda}$ also, define $\tau_{q}: Q_{d} \rightarrow 2^{L}$ by

$$
\tau_{d}\left(Q_{x}\right)=\left\{\begin{array}{ll}
\{\alpha\} & \text { if } q \in Q_{x}, \text { and } \tau(q) \neq\{0\} \\
\{0\} & \text { otherwise }
\end{array},\right.
$$

where $\alpha \in L$. Clearly, $\delta_{d}, \iota_{d}$ and $\tau_{d}$ are well-defined. It is easy to see that $\mathcal{A}=\left(Q_{d}, X, \iota_{d}, \delta_{d}, \tau_{d}\right)$ is a deterministic HLFA. Now, we show that $\mathcal{L}_{\mathcal{A}}=\mathcal{L}_{\mathcal{A}_{d}}$. Let $x \in \mathcal{L}_{\mathcal{A}}$. Then $\sqcup_{p, q \in Q} \iota(p) \sqcap \delta^{*}(p, x, q) \sqcap \tau(q) \neq\{0\}$. So, $\iota(p) \sqcap \delta^{*}(p, x, q) \sqcap \tau(q) \neq\{0\}$, for some $p, q \in Q$. Therefore, $\iota(p) \neq\{0\}, \delta^{*}(p, x, q) \neq\{0\}$ and $\tau(q) \neq\{0\}$. So, $\iota_{d}\left(Q_{\Lambda}\right)=\{\alpha\}, \delta_{d}\left(Q_{\Lambda}, x, Q_{x}\right)=\{\alpha\}$, since $q \in Q_{x}$ and $\tau(q) \neq\{0\}$, so $\tau_{d}\left(Q_{x}\right)=\{\alpha\}$. Therefore, $\iota_{d}\left(Q_{\Lambda}\right) \sqcap \delta_{d}\left(Q_{\Lambda, x, Q_{x}}\right) \sqcap \tau_{d}\left(Q_{x}\right) \neq\{0\}$. So, $x \in \mathcal{L}_{\mathcal{A}_{d}}$. Now, let $x \in \mathcal{L}_{\mathcal{A}_{d}}$. Then $\sqcup_{Q, Q^{\prime} \in Q_{d}} \iota_{d}(Q) \sqcap \delta_{d}\left(Q, x, Q^{\prime}\right) \sqcap$ $\tau_{d}\left(Q^{\prime}\right) \neq\{0\}$. By definition $\iota_{d}$ and $\delta_{d}$ we have

$$
\sqcup_{Q, Q^{\prime} \in Q_{d} \iota_{d}}(Q) \sqcap \delta_{d}\left(Q, x, Q^{\prime}\right) \sqcap \tau_{d}\left(Q^{\prime}\right)=\iota_{d}\left(Q_{\Lambda}\right) \sqcap \delta_{d}\left(Q_{\Lambda}, x, Q_{x}\right) \sqcap \tau_{d}\left(Q_{x}\right) \neq\{0\}
$$

Then $\iota_{d}\left(Q_{\Lambda}\right) \neq\{0\}, \delta_{d}\left(Q_{\Lambda}, x, Q_{x}\right) \neq\{0\}$ and $\tau_{d}\left(Q_{x}\right) \neq\{0\}$. Since $\tau_{d}\left(Q_{x}\right) \neq\{0\}$, then there exists $q^{\prime} \in Q_{x}$ such that $\tau\left(q^{\prime}\right) \neq\{0\}$. By definition $Q_{x}, \iota(p) \sqcap \delta^{*}\left(p, x, q^{\prime}\right) \neq\{0\}$, so $\iota(p) \sqcap \delta^{*}\left(p, x, q^{\prime}\right) \sqcap \tau\left(q^{\prime}\right) \neq\{0\}$. Therefore, $x \in \mathcal{L}_{\mathcal{A}}$. Hence, the claim holds.

Definition 19. Let $\mathcal{L}$ be an HLFL and let $\mathcal{A}$ be an HLFA. We say that $\mathcal{A}$ is minimal HLFA if and only if for every complete accessible deterministic HLFA $\mathcal{A}^{\prime}$ recognizable $\mathcal{L},|\mathcal{A}| \leq\left|\mathcal{A}^{\prime}\right|$.

Definition 20. For every hesitant L-fuzzy language $\mathcal{L} \subseteq X^{*}$ and $u \in X^{*}$, the subset $\mathcal{L}_{u}=\left\{v \in X^{*} \mid u v \in \mathcal{L}\right\}$ of $X^{*}$ is called a right quotient of $\mathcal{L}$ with respect to $u$. 
Let $Q_{\mathcal{L}}=\left\{\mathcal{L}_{u} \mid u \in X^{*}\right\}$ be the set of all right quotient of $\mathcal{L}$ and $\iota_{\mathcal{L}}: Q_{\mathcal{L}} \rightarrow 2^{L}$ defined by $\iota_{\mathcal{L}}\left(\mathcal{L}_{\Lambda}\right)=\{1\}$ and $\iota_{\mathcal{L}}\left(\mathcal{L}_{u}\right)=\{0\}$, where $\mathcal{L}_{u} \neq \mathcal{L}_{\Lambda}$. Also, let $\delta_{\mathcal{L}}: Q_{\mathcal{L}} \times X \times Q_{\mathcal{L}} \rightarrow 2^{L}$ defined by

$$
\delta_{\mathcal{L}}\left(\mathcal{L}_{u}, a, \mathcal{L}_{v}\right)= \begin{cases}\{1\} & \text { if } \mathcal{L}_{u a}=\mathcal{L}_{v} \\ \{0\} & \text { otherwise }\end{cases}
$$

and $\tau_{\mathcal{L}}: Q_{\mathcal{L}} \rightarrow 2^{L}$ defined by

$$
\tau_{\mathcal{L}}\left(\mathcal{L}_{u}\right)=\left\{\begin{array}{ll}
\{1\} & \text { if } u \in \mathcal{L} \\
\{0\} & \text { otherwise }
\end{array},\right.
$$

where $0 \neq \beta \in L$. We show that $\mathcal{A}_{\mathcal{L}}=\left(Q_{\mathcal{L}}, X, \iota_{\mathcal{L}}, \delta_{\mathcal{L}}, \tau_{\mathcal{L}}\right)$ is a finite HLFA.

Lemma 1. Let $\mathcal{L} \subseteq X^{*}$ and $Q_{\mathcal{L}}$ be a finite set. Then $\mathcal{L}$ is a recognizable hesitant $L$-fuzzy language.

Proof. Let $\mathcal{A}_{\mathcal{L}}=\left(Q_{\mathcal{L}}, X, \iota_{\mathcal{L}}, \delta_{\mathcal{L}}, \tau_{\mathcal{L}}\right)$ be an HLFA. We show that $\mathcal{L}_{\mathcal{A}_{\mathcal{L}}}=\mathcal{L}$. If $w \in \mathcal{L}$, then $\tau_{\mathcal{L}}\left(\mathcal{L}_{w}\right)=\{1\}$. Also, $\delta_{\mathcal{L}}\left(\mathcal{L}_{\Lambda}, w, \mathcal{L}_{w}\right)=\{1\}$ and by considering definition $\iota_{\mathcal{L}}$ we have $\iota_{\mathcal{L}}\left(\mathcal{L}_{\Lambda}\right) \sqcap \delta_{\mathcal{L}}\left(\mathcal{L}_{\Lambda}, w, \mathcal{L}_{w}\right) \sqcap \tau_{\mathcal{L}}\left(\mathcal{L}_{w}\right)=\{1\}$. Then $\sqcup_{p, q \in Q_{\mathcal{L}}} \iota_{\mathcal{L}}(p) \sqcap \delta_{\mathcal{L}}(p, w, q) \sqcap \tau_{\mathcal{L}}(q) \neq\{0\}$. Hence, $w \in \mathcal{L}\left(\mathcal{A}_{\mathcal{L}}\right)$. Now, let $w \in \mathcal{L}\left(\mathcal{A}_{\mathcal{L}}\right)$. By definition of $\iota_{\mathcal{L}}$ we have

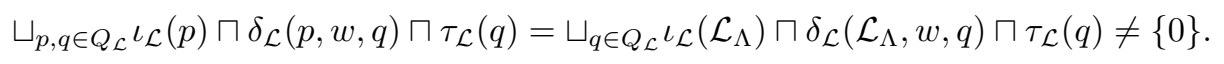

Now, by considering definitions $\delta_{\mathcal{L}}$ and $\tau_{\mathcal{L}}$, we have $q=\mathcal{L}_{w}$ and $\tau_{\mathcal{L}}\left(\mathcal{L}_{w}\right) \neq\{0\}$. Then $\tau_{\mathcal{L}}\left(\mathcal{L}_{w}\right) \neq\{0\}$ implies that $w \in \mathcal{L}$. Hence, $\mathcal{L}\left(\mathcal{A}_{\mathcal{L}}\right)=\mathcal{L}$.

Theorem 9. Let $\mathcal{L}$ be a recognizable hesitant L-fuzzy language. Then $\mathcal{A}_{\mathcal{L}}=\left(Q_{\mathcal{L}}, X, \iota_{\mathcal{L}}, \delta_{\mathcal{L}}, \tau_{\mathcal{L}}\right)$ is a minimal HLFA.

Proof. Let $\mathcal{L}$ be a recognizable HLFL. Then there exists a complete, accessible deterministic HLFA $\mathcal{A}=(Q, X, \iota, \delta$, $\tau)$ such that $\iota\left(q_{0}\right) \neq\{0\}$ and $\mathcal{L}\left(\mathcal{A}_{\mathcal{L}}\right)=\mathcal{L}$. Define a map $\varphi: Q \rightarrow Q_{\mathcal{L}}$ by $\varphi(p)=\mathcal{L}_{u}$, where $\delta^{*}\left(q_{0}, u, p\right) \neq\{0\}$. Clearly, $\varphi$ is well-defined and surjective so, $\left|Q_{\mathcal{L}}\right| \leq|Q|$ and $Q_{\mathcal{L}}$ is finite. Hence, $\mathcal{A}_{\mathcal{L}}$ is a minimal HLFA.

The following algorithm determines the states of minimal automaton.

Algorithm1: (Determines the states of minimal hesitant L-fuzzy automaton)

Step 1. input: accessible deterministic $\operatorname{HLFA}, \mathcal{A}=(Q, X, \iota, \delta, \tau)$,

Step 2. $X_{l}=\left\{x \in X^{*}|| x \mid=l\right\}$, let $l=0$,

Step 3. $q_{1} \kappa_{l} q_{2}$ if and only if $\tau\left(q_{1}\right) \neq\{0\} \Leftrightarrow \tau\left(q_{2}\right) \neq\{0\}$,

Step 4. $l=l+1$,

Step 5. $q_{1} \kappa_{l} q_{2}$ if and only if $q_{1} \kappa_{l-1} q_{2}$ and

$$
\left\{w \in X^{*}\left|\delta\left(q_{1}, w, q\right) \sqcap \tau(q) \neq\{0\},\right| w \mid=l\right\}=\left\{w \in X^{*}\left|\delta\left(q_{2}, w, q\right) \sqcap \tau(q) \neq\{0\},\right| w \mid=l\right\},
$$


Step 6. if $\kappa_{l+1}=\kappa_{l}$, go to next step,

else go to Step 4,

Step 7. $\kappa=\kappa_{l}$,

Step 8. output: $\kappa$

Now, let $q_{1} \kappa q_{2}$, since $\mathcal{A}$ is accessible and deterministic, then there exist $u, v \in X^{*}$ such that $\delta\left(q_{0}, u, q_{1}\right) \neq\{0\}$ and $\delta\left(q_{0}, u, q_{2}\right) \neq\{0\}$. Hence, by considering Algorithm $1, \mathcal{L}_{u}=\mathcal{L}_{v}$.

Steps 4 to 6 of Algorithm 1, are a loop. The loop must be repeated at most $O(|Q|)$ times. The order of time complexity to calculate $l=0$ is $O\left(|Q|^{2}\right)$, to calculate $l=1$ is $O\left(|Q|^{2}|X|\right)$, to calculate $l=2$ is $O\left(|Q|^{2}|X|^{2}\right)$. Then the time complexity of calculating this algorithm is $O\left(|Q|^{2}|X|^{|Q|}\right)$.

\section{Conclusion}

In this paper, we present the theory of hesitant L-fuzzy automaton and hesitant L-fuzzy behavior and hesitant Lfuzzy language. We present some of the closure properties for the HLFA such as union and complement. For any hesitant L-fuzzy language $A$, we present a minimal complete accessible deterministic hesitant L-fuzzy automaton recognizing $A$. Also, we give an algorithm to determines states of the minimal hesitant L-fuzzy automaton.

Acknowledgement No funding was received.

Compliance with ethical standards

Conflict of interest The authors declare that they have no conflict of interest.

Ethical approval This article does not contain any studies with human participants or animals performed by any of the authors.

Informed consent Informed consent was obtained from all individual participants included in the study.

Authors' contributions All authors (M. Shamsizadeh, M. M. Zahedi, Kh, Abolpour) whose names appear on the submission

1. made substantial contributions to the conception or design of the work; or the acquisition, analysis, or interpretation of data; or the creation of new software used in the work,

2. drafted the work or revised it critically for important intellectual content,

3. approved the version to be published; and

4. agree to be accountable for all aspects of the work in ensuring that questions related to the accuracy or integrity of any part of the work are appropriately investigated and resolved.

\section{References}

[1] N.C. Basak, A.Gupta, On quotient machines of a fuzzy automata and the minimal machine, Fuzzy Sets and Systems, 125 (2002), 223-229. 
[2] B. Bedregal, R. Reiser, H. Bustince, C. Lopez-Molina, V. Torra, Aggregation functions for typical hesitant fuzzy elements and the action of automorphisms. Information Sciences, 2014. 255:82-99.

[3] W. Cheng, Z.W. Mo, Minimization algorithm of fuzzy finite automata, Fuzzy Sets and Systems, 141 (2004), 439-448.

[4] VS. Costa, B. Bedregal, On typical hesitant fuzzy automata. Soft Computing, 2020. 24(12):8725-8736.

[5] A. H. Dehmiry, M. Mashinchi, R. Mesiar, Hesitant L-Fuzzy Sets, International journal of intelligent systems, 33 (2018), 1027-1042.

[6] J. M. Howie, Automata and languages. Oxford University Press, Inc., 1992.

[7] Y. B. Jun, Quotient structures of intuitionistic fuzzy finite state machines, Information Sciences, 177 (2007), 4977-4986.

[8] H.S. Lee, Minimizing fuzzy finite automata, 2000. Fuzzy Systems, FUZZ IEEE 2000. The Ninth IEEE International Conference on, 1 (2000), 65-70.

[9] E. T. Lee, L. A. Zadeh, Note on fuzzy languages, Information Sciences, 1 (1969), 421-434.

[10] D.S. Malik and J.N. Mordeson, Fuzzy Automata and Languages: Theory and Applications, Chapman Hall, CRC Boca Raton, London, New York, Washington DC, 2002.

[11] D. S. Malik, J.N. Mordeson, Fuzzy Discrete Structures, Physica-Verlag, New York, 2000, London, 2002.

[12] A. Mateescu, A. Salomaa, K. Salomaa and S. Yu, Lexical Analysis with a Simple Finite Fuzzy Automaton Model, Journal of Universal Computer Science, 1 (1995), 292-311.

[13] D. Qiu, Supervisory control of fuzzy discrete event systems: a formal approach, IEEE Transactions on Systems, Man and CyberneticsPart B, 35 (2005), 72-88.

[14] M. Shamsizadeh, M. M. Zahedi, Bisimulation of type 2 for BL-general fuzzy automata, Soft Computing, 23 (2019), 9843-9852.

[15] M. Shamsizadeh, M. M. Zahedi, Intuitionistic general fuzzy automata, Soft Computing, 20 (2016), 35053519.

[16] A. K. Srivastava, S. P. Tiwari, IF-topologies and IF-automata, Soft Computing, 14 (2010), 571-578.

[17] S. P. Tiwari, Sh. Sharan, Fuzzy automata based on lattice-ordered monoid with algebraic and topological aspects, Fuzzy information and engineering, 4 (2012), 155-164.

[18] S. P. Tiwari, A. K. Srivastava, On a decomposition of fuzzy automata, Fuzzy Sets and Systems, 151 (2005), 503-511.

[19] V. Torra, Hesitant fuzzy sets, International Journal of Intelligent Systems 25.6 (2010): 529-539.

[20] V. Torra, Y. Narukawa, On hesitant fuzzy sets and decision, 2009 IEEE International Conference on Fuzzy Systems. IEEE, 2009. 
[21] W. G. Wee, On generalization of adaptive algorithm and application of the fuzzy sets concept to pattern classification, Ph.D. Thesis, Purdue University, Lafayette, IN, 1967.

[22] L. A. Zadeh, Fuzzy sets, Information and Control, 8 (1965,) 338-353

Publishers Note Springer Nature remains neutral with regard to jurisdictional claims in published maps and institutional affiliations. 